Pacific Journal of Mathematic 


\section{ALGORITHMS FOR LOCALIZING ROOTS OF A POLYNOMIAL AND THE PISOT VIJAYARAGHAVAN NUMBERS}

\section{R. J. DUFFIN}

Pisot and Vijayaraghavan studied numbers whose $m$ th power is nearly an integer in the sense that the discrepancy vanishes as $m$ becomes infinite. One plus square root two is an example. Algebraic numbers of this type are characterized as algebraic integers whose conjugate roots each have absolute value less than one. This note develops a test for this property. An algorithm is given which determines whether or not one root of a polynomial has absolute value greater than one and all the other roots have absolute value less than one. If $n$ is the degree of the polynomial, this algorithm involves only $n$ rational steps.

1. Introduction. In a previous paper [2] the writer formulated a simple algorithm for testing a polynomial to see if all of its roots were in the interior of the unit circle. Polynomials of this type were termed Schur polynomials. The importance of such polynomials rests on the fact that a linear difference equation with constant coefficients is stable if and only if the characteristic polynomial is of Schur type. The test given in [2] follows.

Algorithm $\mathrm{A}_{0}$. Let $f$ be the polynomial

$$
f(z)=c_{0}+c_{1} z+c_{2} z^{2}+\cdots+c_{n} z^{n}
$$

where $c_{n} \neq 0$ and $n \geqq 1$. Let $R f$ be a polynomial of reduced degree defined with $c_{j}^{*}$ the complex conjugate of $c_{j}$ as

$$
R f(z)=\left(c_{n}^{*} c_{1}-c_{0} c_{n-1}^{*}\right)+\left(c_{n}^{*} c_{2}-c_{0} c_{n-2}^{*}\right) z+\cdots+\left(c_{n}^{*} c_{n}-c_{0} c_{0}^{*}\right) z^{n-1} .
$$

Then $f$ is a Schur polynomial if and only if:

(i) $\left|c_{n}\right|>\left|c_{0}\right|$,

(ii) Rf is a Schur polynomial.

In a series of papers John Miller [4, 5] has extended this algorithmic test for Schur polynomials. Miller's tests determine the "type" of a polynomial, that is, the number of roots inside, on, and outside the unit circle.

The present note was prompted by the works of Pisot and Vijayaraghavan about what may be termed powerful numbers $[1,6,7]$. A number greater than one is powerful if its $m$ th power 
is nearly an integer in the sense that the discrepancy vanishes as $m$ becomes infinite. Common examples are $1+\sqrt{2}$ and $(1+\sqrt{5}) / 2$. Pisot and Vijayaraghavan showed that an algebraic number $\alpha$ is powerful if and only if $\alpha$ is an algebraic integer whose conjugate roots have absolute value less than one. Thus $\alpha$ must satisfy an equation

$$
f(\alpha)=b_{0}+b_{1} \alpha+\cdots+b_{n-1} \alpha^{n-1}+\alpha^{n}=0 .
$$

where $b_{0}, b_{1}, \cdots, b_{n-1}$ are rational integers.

This note develops an algorithm to test a polynomial of degree $n$ for the Pisot Vijayaraghavan property. The method is to first apply a reduction operation which removes one root exterior to the unit circle. Then Algorithm $A_{0}$ is applied to the reduced polynomial to see if it has $n-1$ roots interior to the unit circle. Only integer arithmetic is used.

The writer is indebted to Paul Bateman and David Cantor for discussions about the Pisot Vijayaraghavan theory. One part of $\S 4$ to follow employs a technique developed by John Miller. An alternative procedure equivalent to $\S 2$ is given by Peter Henrici [3, p. 494]. No confusion should arise with the terminology of S. W. Golomb, who defined a "powerful integer" as an integer divisible by the square of each of its prime divisors [Amer. Math. Mon. 77 (1970) pp. 848-855].

2. Two root localizing algorithms. Passing to a more general problem we shall say that a polynomial is of type $(i, k)$ if:

(a) $i$ roots are in the interior of the unit circle,

(b) no roots are on the unit circle,

(c) $k$ roots are in the exterior of the unit circle.

First Algorithm $A_{0}$ is generalized to

Algorithm A. Let $f$ be the polynomial

$$
f(z)=c_{0}+c_{1} z+\cdots+c_{n} z^{n}, \quad n \geqq 1, \quad c_{n} \neq 0,
$$

and let $R f$ be a polynomial of reduced degree defined as

$$
R f(z)=\left(c_{n}^{*} c_{1}-c_{0} c_{n-1}^{*}\right)+\left(c_{n}^{*} c_{2}-c_{0} c_{n-2}^{*}\right) z+\cdots+\left(c_{n}^{*} c_{n}-c_{0} c_{0}^{*}\right) z^{n-1} .
$$

If $\left|\boldsymbol{c}_{n}\right|>\left|\boldsymbol{c}_{0}\right|$ then $f$ is of type $(i, k)$ if and only if $R f$ is of type $(i-1, k)$.

Proof. Given the polynomial $f$ of degree $n$ let

$$
J f(z)=c_{n}^{*}+c_{n-1}^{*} z+\cdots+c_{0}^{*} z^{n}=z^{n} f^{*}\left(z^{-1}\right) .
$$

The operation $J$ depends, of course, on the stated value of $n$ because 
a polynomial of degree $n$ is also of degree $n+1$. Then operation $R$ may be defined as

$$
z R f(z)=c_{n}^{*} f(z)-c_{0} J f(z) .
$$

First suppose that $f$ is of type $(i, k)$. Then for $n=i+k$ and $|z|=1$

$$
\left|c_{n}^{*} f(z)\right|>\left|c_{0}^{*} f(z)\right|=\left|c_{0} f^{*}\left(z^{*}\right)\right|=\left|c_{0} J f(z)\right| .
$$

The strict inequality here is justified by the fact that $f$ does not vanish on the circle. Inequality (4) permits application of Rouche's theorem. Thus $z R f$ and $c_{n}^{*} f$ have the same number of roots for $|z|<1$; moreover $z R f$ has no roots for $|z|=1$. Hence $R f$ has $i-1$ roots for $|z|<1$.

Since $\left|c_{n}\right|>\left|c_{0}\right|$ it is seen from (2) that the leading coefficient of $R f$ does not vanish. Thus $R f$ has a total of $n-1$ roots. But $i+$ $k=n$ so $R f$ is of type $(i-1, k)$.

Conversely suppose $R f$ is of type $(i-1, k)$. If $f\left(z^{\prime}\right)=0$ at a point $z^{\prime}$ on the unit circle then $J f\left(z^{\prime}\right)=0$ because $|f(z)| \equiv|J f(z)|$ on the unit circle. Then (3) would demand that $R f\left(z^{\prime}\right)=0$. This contradiction shows that $f$ can not vanish on the unit circle. Thus the inequality (4) again holds and it again follows from Rouche's theorem that $f$ and $z R f$ are both of type $(i, k)$. This is seen to complete the proof.

Algorithm B. Let $f$ be the polynomial

$$
f(z)=c_{0}+c_{1} z+\cdots+c_{n} z^{n}, \quad n \geqq 1
$$

and let $S f$ be a polynomial of reduced degree defined as

$$
S f(z)=\left(c_{n} c_{n}^{*}-c_{0}^{*} c_{0}\right)+\left(c_{n} c_{n-1}^{*}-c_{0}^{*} c_{1}\right) z+\cdots+\left(c_{n} c_{1}^{*}-c_{0}^{*} c_{n-1}\right) z^{n-1} .
$$

If $\left|c_{0}\right|>\left|c_{n}\right|>0$ then $f$ is of type $(i, k)$ if and only if $S f$ is of type $\left(i, k^{\prime}\right)$ where $i+k^{\prime}$ is the minimum degree of $S f$.

Proof. This is a corollary of Algorithm A. To see this let $F=J f$. Then $F$ is of the form

$$
F(z)=C_{0}+C_{1} z+\cdots+C_{n} z^{n}, \quad\left|C_{n}\right|>\left|C_{0}\right|>0 .
$$

Moreover the zeros of $f$ and $F$ are inverse points relative to the unit circle. Thus $f$ is of type $(i, k)$ if and only if $F$ is of type $(k, i)$. Applying Algorithm $\mathrm{A}$ to $\mathrm{F}$ shows that $f$ is of type $(i, k)$ if and only if $R F$ is of type $(k-1, i)$. Inspection of (2) and (5) shows that $J R F=S F$. Hence $R F$ is of type $(k-1, i)$ if and only if $S F$ is 
of type $\left(i, k^{\prime}\right)$. Of course $k^{\prime}=k-1$ if $c_{n} c_{1}^{*}-c_{0}^{*} c_{n-1} \neq 0$. Otherwise $k^{\prime}<k-1$, and the proof is seen to be complete.

3. Searching for powerful numbers. Now of concern is the special case of polynomials of type $(n-1,1)$. Algorithm B specializes to the following form

Algorithm $\mathrm{B}_{1}$. If $\left|c_{0}\right|>\left|c_{n}\right|>0$ then $f$ is of type $(n-1,1)$ if and only if $S f$ is of type $(n-1,0)$.

Of course a polynomial of type $(n-1,0)$ is a Schur polynomial. Hence if $f$ passes the test of Algorithm $\mathrm{B}_{1}$ the next step is apply Algorithm $\mathrm{A}_{0}$ to $S f$ etc.

There follows an example of the use of these algorithms to show that a polynomial $f$ is of type $(3,1)$.

$$
\begin{aligned}
f & =2+z-z^{2}-4 z^{3}+z^{4}, \\
S f & =-3-6 z+z^{2}+9 z^{3}, \\
R S f & =-51-9 z+72 z^{2}, \\
R R S f & =-1107+2583 z .
\end{aligned}
$$

Thus $R R S f$ is of type $(1,0), R S f$ is of type $(2,0)$, and $S f$ is of type $(3,0)$. Thus $f$ has three roots inside the unit circle and one root outside. Also $f(1)<0$ so it is apparent that $f$ has a positive root $\alpha>1$. Hence $\alpha$ is a powerful number.

To give a numerical evaluation of $\alpha$ and its powers it is relevant to introduce the Newton sequence $\left\{S_{m}\right\}$ defined as

$$
S_{m}=\alpha^{m}+\alpha_{1}^{m}+\alpha_{2}^{m}+\alpha_{3}^{m}
$$

where $\alpha_{1}, \alpha_{2}$ and $\alpha_{3}$ are the conjugate roots of $\alpha$. As Newton showed:

$$
\begin{aligned}
& -s_{1}=b_{3}, \\
& -s_{2}=s_{1} b_{3}+2 b_{2}, \\
& -s_{3}=s_{2} b_{3}+s_{1} b_{2}+3 b_{1}, \\
& -s_{4}=s_{3} b_{3}+s_{2} b_{2}+s_{1} b_{1}+4 b_{0} .
\end{aligned}
$$

Moreover for larger values of $m$ the Newton sequence satisfies the difference equation

$$
-s_{m+4}=s_{m+3} b_{3}+s_{m+2} b_{2}+s_{m+1} b_{1}+s_{m} b_{0} .
$$

Thus for $m=1,2, \cdots$

$$
\left\{s_{m}\right\}=4,18,73,298,1239,5145,21375,88810, \cdots \text {. }
$$


It is clear that $\alpha=\lim s_{m+1} / s_{m}$ so $\alpha=4.154869708$. Moreover $\alpha^{4} \approx$ $298, \alpha^{5} \approx 1239$, etc.

4. A special algorithm. Algorithms $A$ and $B$ assume either $\left|c_{n}\right|>\left|c_{0}\right|$ or $\left|c_{0}\right|>\left|c_{n}\right|$. Here we assume $\left|c_{0}\right|=\left|c_{n}\right|$ and develop a special algorithm to test powerful polynomials.

Lemma 1. If $\left|c_{0}\right|=\left|c_{n}\right|>0$ and $f$ is of type $(n-1,1)$ with $n>2$ then $c_{n} c_{1}^{*}-c_{0}^{*} c_{n-1} \neq 0$.

Proof. Let $f_{\theta}(z)=f(\theta z)$ so

$$
f_{\theta}(z)=c_{0}+c_{1} \theta z+\cdots+c_{n} \theta^{n} z^{n} .
$$

By continuity it follows that if $\varepsilon$ is a sufficiently small positive number and $\theta$ is in the range $1-\varepsilon<\theta<1$ then $f_{\theta}$ is also of the type $(n-1,1)$. However Algorithm $B_{1}$ applies to $f_{\theta}$. Thus $S f_{\theta}$ has all $n-1$ roots interior to the unit circle. As $\theta \rightarrow 1$ we see that $S f_{\theta} \rightarrow S f$. By a well known lemma of Hurwitz it follows that the roots of $S f_{\theta}$ approach the roots of $S f$. Thus $S f$ has at least $n-1$ roots in the closed circle $|z| \leqq 1$.

Conceivably $S f \equiv 0$ but from (5) it is seen that

$$
S f=-c_{0}^{*} f+c_{n} J f .
$$

Hence if $f\left(z^{\prime}\right)=0$ we have $f\left(z^{\prime \prime}\right)=0$ where $z^{\prime}$ and $z^{\prime \prime}$ are inverse points. But there is only one root for $|z|>1$ so there is only one root for $0<|z|<1$. However $f(z) \neq 0$ for $z=0$ or $|z|=1$ so $f$ is of degree 2. This contradiction shows that $S f$ does not vanish identically and that $S f$ has $n-1$ roots. Thus the leading coefficient $c_{n} c_{1}^{*}-c_{0}^{*} c_{n-1}$ does not vanish.

Given a polynomial $f$ of degree $n$ let a polynomial $T f$ of degree $n+1$ be defined for a real constant $\beta$ as

$$
T f(z)=(z+\beta) f(z), \quad|\beta|>1 .
$$

Then $f$ is of type $(n-1,1)$ if and only if $T f$ is of type $(n-1,2)$. Of course

$$
\begin{aligned}
T f & =\beta c_{0}+\left(c_{0}+\beta c_{1}\right) z+\cdots+\left(c_{n-1}+\beta c_{n}\right) z^{n}+c_{n} z^{n+1}, \\
J T f & =z^{n+1}\left(z^{-1}+\beta\right) f^{*}\left(z^{-1}\right)=(1+\beta z) J f .
\end{aligned}
$$

Since $S f=-c_{0}^{*} f+c_{n} J f$ we see that

$$
\begin{aligned}
S T f & =-\beta c_{0}^{*} T f+c_{n} J T f \\
& =-\beta c_{0}^{*}(z+\beta) f+c_{n}(1+\beta z) J f
\end{aligned}
$$




$$
\begin{aligned}
& =-\beta^{2} c_{0}^{*} f+c_{n} J f+\beta z\left(-c_{0}^{*} f+c_{n} J f\right) \\
& =-\beta^{2} c_{0}^{*} f+c_{n} J f+\beta z S f .
\end{aligned}
$$

Expressing the right side in powers of $z$ gives

$$
\begin{aligned}
S T f= & \left(c_{n} c_{n}^{*}-\beta^{2} c_{0}^{*} c_{0}\right)+\cdots+\left(1-\beta^{2}\right) c_{n} c_{0}^{*} z^{n} \\
& +\beta\left[\left(c_{n} c_{n}^{*}-c_{0}^{*} c_{0}\right) z+\cdots+\left(c_{n} c_{1}^{*}-c_{0}^{*} c_{n-1}\right) z^{n}\right] .
\end{aligned}
$$

LeMmA 2. Suppose $\left|c_{0}\right|=\left|c_{n}\right|>0$ and that $c_{n} c_{1}^{*}-c_{0}^{*} c_{n-1} \neq 0$. Let $T f=(z+\beta) f$ where $|\beta|>1$ is chosen so that

$$
c_{n}^{\prime}=\beta\left(c_{n} c_{1}^{*}-c_{0}^{*} c_{n-1}\right)+\left(1-\beta^{2}\right) c_{n} c_{0}^{*} \neq 0 .
$$

Then $f$ is of type $(n-1,1)$ if and only if STf is of type $(n-1,1)$.

Proof. The constant coefficient of $T f$ is $\beta c_{0}$ and the coefficient of $z^{n+1}$ is $c_{n}$. Since $\left|\beta c_{0}\right|>\left|c_{n}\right|$ Algorithm B is applicable to Tf. The coefficient of $z^{n}$ of $S T f$ is $c_{n}^{\prime}$ given by (6). But $c_{n}^{\prime} \neq 0$ so $S T f$ has $n$ roots. Thus $T f$ is of type $(n-1,2)$ if and only if $S T f$ is of type $(n-1,1)$. This is seen to complete the proof.

Algorithm C. Let $f$ be a polynomial of degree $n>2$ and with real coefficients such that $\left|c_{0}\right|=\left|c_{n}\right|>0$. Let $T f(z)=(z+\beta) f(z)$ for $\beta$ chosen so that

$$
\beta^{2}-1>\left|\beta^{2}-1-\beta d\right|>0
$$

where

$$
d=\left(c_{n} c_{1}-c_{0} c_{n-1}\right) / c_{n} c_{0} .
$$

Then $f$ is of type $(n-1,1)$ if and only if:

(i) $c_{n} c_{1}-c_{0} c_{n-1} \neq 0$,

(ii) $S S T f$ is of type $(n-1,0)$.

Proof. If $f$ is of type $(n-1,1)$ then Lemma 1 proves relation (i). Since (i) holds it follows that $d \neq 0$. It is then obvious that $\beta$ can be chosen to satisfy (7). Thus Lemma 2 applies because of relation (i) and the fact that if $\beta$ satisfies (7) then condition (6) holds. Lemma 2 states that $S T f$ is of type $(n-1,1)$. The constant coefficient of $S T f$ is

$$
c_{0}^{\prime}=c_{n} c_{n}-\beta^{2} c_{0} c_{0}=\left(1-\beta^{2}\right) c_{0}^{2} .
$$

To apply Algorithm $\mathrm{B}_{1}$ to $S T f$ we must have

$$
\left|c_{0}^{\prime}\right|>\left|c_{n}^{\prime}\right|>0 \text {. }
$$


In other words

$$
\left(\beta^{2}-1\right) c_{0}^{2}>\left|\beta\left(c_{n} c_{1}-c_{0} c_{n-1}\right)+\left(1-\beta^{2}\right) c_{n} c_{0}\right|>0 .
$$

Dividing by $\left|c_{n} c_{0}\right|$ gives the equivalent relation (7). Thus Algorithm $\mathrm{B}_{1}$ applies and shows that $S S T f$ is of type $(n-1,0)$.

Conversely suppose (i) holds and that SSTf is of type $(n-1,0)$ for some $\beta$ which satisfies (7). Then (9) holds and Algorithm $B_{1}$ shows that $S T f$ is of type $(n-1,1)$. Lemma 2 then proves $f$ is of type $(n-1,1)$.

5. An example of a powerful unit. In the search for powerful numbers attention can be restricted to the case $\left|c_{0}\right| \geqq\left|c_{n}\right|$ because $c_{n}=1$ and $c_{0}$ is an integer. If $\left|c_{0}\right|>\left|c_{n}\right|$ we have seen that Algorithm $\mathrm{B}_{1}$ applies. The case $\left|c_{0}\right|=\left|c_{n}\right|=1$ is now to be treated. An algebraic integer is said to be a unit if its reciprocal is also an integer. It is seen therefore that we are searching for a powerful number which is a unit.

To apply Algorithm $\mathrm{C}$ it is necessary to choose $\beta$ so as to satisfy (7). Since the coefficients are integers it is seen that $d$ is an integer. It is convenient then to choose $\beta$ an integer according to the following rule.

Rule. Choose $\beta$ of the same sign as $d$. If $|d| \leqq 3$ let $|\beta|=$ $|d|+1$, otherwise let $|\beta|=|d|-1$.

It may be checked that this rule insures that inequality (7) is satisfied. Various other rules are also feasible.

As an example consider the polynomial

$$
f=1+z-z^{2}-3 z^{3}+z^{4} .
$$

To apply Algorithm $\mathrm{C}$ first note that $d=c_{1} / c_{0}-c_{3} / c_{4}=4$. So choose $\beta=3$ and $T f=(z+3) f$ so

$$
\begin{aligned}
T f & =3+4 z-2 z^{2}-10 z^{3}+0+z^{5} \\
J T f & =1+0-10 z^{2}-2 z^{3}+4 z^{4}+3 z^{5} \\
S T f & =-2-3 z-z^{2}+7 z^{3}+z^{4}, \quad \div 4 \\
J S T f & =1+7 z-z^{2}-3 z^{3}+2 z^{4} \\
S^{2} T f & =-3+z-3 z^{2}+11 z^{3} \\
J S^{2} T f & =11-3 z+z^{2}-3 z^{3} \\
R S^{2} T f & =1-15 z+56 z^{2}, \quad \div 2 \\
J R S^{2} T f & =56-15 z+z^{2} \\
R^{2} S^{2} T f & =-825+3135 z \quad \text { type }(1,0) .
\end{aligned}
$$


This application of Algorithms $\mathrm{C}$ and $\mathrm{A}_{0}$ shows that $f$ has a root $\alpha$ which is a powerful unit.

The difference equation associated with the polynomial $f$ is

$$
-s_{m+4}=-3 s_{m+3}-s_{m+2}+s_{m+1}+s_{m},
$$

Newton's formulas give:

$$
\begin{aligned}
& -s_{1}=-3 \\
& -s_{2}=-3 s_{1}-2 \\
& -s_{3}=-3 s_{2}-s_{1}+3 \\
& -s_{4}=-3 s_{3}-s_{2}+s_{1}+4 .
\end{aligned}
$$

Thus Newton's series is for $m=1,2, \cdots$,

$$
\left\{s_{m}\right\}=3,11,33,103,328,1043,3321,10575, \cdots \text {. }
$$

Then $\alpha=\lim s_{m+1} / s_{m}=3.184446857$ and $\alpha^{4} \approx 103, \alpha^{5} \approx 328, \alpha^{6} \approx 1043$ etc.

6. Crude algorithms. The tests for polynomial type just given are both necessary and sufficient but always require $n$ steps. The next algorithm gives a sufficient condition for a polynomial to be of type $(i, k)$. In many cases this test requires only one or two steps. (This test, presumably, is not new, but it serves to complete the picture.)

Algorithm D. Let $f$ be the polynomial

$$
f(z)=c_{0}+c_{1} z+\cdots+c_{n} z^{n}, \quad c_{n} \neq 0 .
$$

Then $f$ is of type $(i, n-i)$ if

$$
2\left|c_{i}\right|-\sum_{0}^{n}\left|c_{j}\right|>0
$$

Proof. Write $f=f_{1}+f_{2}$ where $f_{1}=c_{i} z^{i}$ and $f_{2}=f-c_{i} z^{i}$. Thus condition (11) shows that $\left|f_{1}\right|>\left|f_{2}\right|$ on the unit circle. It follows from Rouche's theorem that $f$ has the same number of roots in the interior of the unit circle as $f_{1}$.

Algorithm D'. Again $f$ is of type $(i, n-i)$ if

$$
2\left|c_{i}\right|-\sum_{0}^{n}\left|c_{j}\right|=0
$$

provided $f \neq 0$ on the unit circle. 
Proof. Equality (12) can be treated as a limit of inequality (11). By the lemma of Hurwitz it follows that the limit polynomial $f$ has $i$ roots for $|z|<1$ because $f$ does not vanish for $|z|=1$.

COROLlaRy 1. The polynomial $f=-1-b z^{n-1}+z^{n}$ has a powerful root if $b$ is an integer $\geqq 2$.

Proof. Note that $f(1)<0$ and that $f(x)>0$ for large positive $x$. Thus $f$ has a root $>1$. If $b>2$ this corollary follows directly from Algorithm D. If $b=2$ and $|z|=1$ then $\left|z^{n}-2 z^{n-1}\right|=1$ implies $z=1$ but $f(1)=-2$. Hence $f(z) \neq 0$ so Algorithm $\mathrm{D}^{\prime}$ applies to complete the proof.

It is of interest to consider whether Corollary 1 holds for $b=1$. Thus if $n=2$ then $f=-1-z+z^{2}$ has the powerful root

$$
\frac{1+\sqrt{5}}{2}=1.618033989 \text {. }
$$

Of course this is the golden ratio and is the smallest quadratic powerful number. This suggests $f=-1-z+z^{3}$, and Cardan's formula gives the powerful root

$$
\left[\frac{1^{\prime}}{2}+\frac{(69)^{1 / 2}}{18}\right]^{1 / 3}+\left[\frac{1}{2}-\frac{(69)^{1 / 2}}{18}\right]^{1 / 3}=1.324717957 .
$$

This is an analog of the golden ratio for the cubic domain. Presumably it is the smallest cubic powerful number.

Next consider $n=5$ and $f=-1-z^{4}+z^{5}$. To apply Algorithm C we note that $d=1$ and $\beta=2$ so $T f=(z+2) f$. Thus:

$$
\begin{aligned}
T f & =-2-z-2 z^{4}+z^{5}+z^{6} \\
S T f & =3+z+2 z^{2}+4 z^{5}-z^{5} \\
S^{2} T f & =8+7 z+6 z^{2}+2 z^{3}+13 z^{4} \\
R S^{2} T f & =5+2 z-2 z^{2}+7 z^{3}, \quad \div 15 \\
R^{2} S^{2} T f & =1-z+z^{2}, \quad \div 24, \text { type }(1,1) .
\end{aligned}
$$

It is thereby seen that $f$ is of type $(3,2)$ so Corollary 1 is not generally valid for $b=1$.

Corollary 2. Every rational integer $\geqq 2$ is a limit point of powerful units.

Proof. Corollary 1 shows that there is a powerful unit $\alpha_{n}$ such that $\alpha_{n}=b+1 / \alpha_{n}^{n-1}$. Thus $b<\alpha_{n}$ and so

$$
b<\alpha_{n}<b+1 / b^{n-1} .
$$


Allowing $n \rightarrow \infty$ we see that $\alpha_{n} \rightarrow b$.

The short cut afforded by Algorithm $D$ can be seen from the calculations given for the example of $\S 5$. First note that Algorithm D applies to $S^{2} T f$ because $-3-1-3+11>0$ and relation (11) is satisfied for $i=3$. Thus $S^{2} T f$ is of type $(3,0)$ and further steps in the calculation are not needed.

A still shorter solution is obtained by noting that for $T f$ relation (12) with $i=3$ is $-3-4-2+10-0-1=0$. Moreover it is clear that $T f$ can not vanish if $|z|=1$. Thus Algorithm $\mathrm{D}^{\prime}$ applies to show that $T f$ is of type $(3,2)$. Of course this means that $f$ is of type $(3,1)$.

It is also possible to give necessary conditions for a polynomial to be of type $(i, k)$. One such test was given in [2].

Algorithm E. If $f$ is of type $(n, 0)$ then

$$
\left|c_{j}\right|<\left(\begin{array}{c}
n \\
j
\end{array}\right)\left|c_{n}\right|, \quad j=0,1, \cdots, n-1 .
$$

Various other crude tests may be developed.

\section{REFERENCES}

1. J. W. S. Cassels, An Introduction to Diophantine Approximation, Cambridge Tract No. 45, Chap. VIII, Cambridge, 1957.

2. R. J. Duffin, Algorithms for classical stability problems, SIAM Review, 11 (1969), 196-213.

3. Peter Henrici, Applied and Computational Complex Analysis, Vol. I. John Wiley, New York, 1974.

4. John J. H. Miller, On the stability of differential equations, SIAM J. Control, 10 (1972), 639-648.

5. - Practical alggrithms for finding the type of a polynomial, Lanczos Festschrift, Academic Press, 1973.

6. Ch. Pisot, Eine merkwürdige Klasse ganzer algebrischer Zahlen, J. f.d. reine u. angew. Mathematik, 209 (1962), 82-83.

7. — Quelques Aspects de la Théorie des Entiers Algébriques, 2nd Edition, Universite de Montréal, 1966.

Received November 15, 1976 and in revised form August 9, 1977. Supported by Grant DAAG29-77-G-0024 of the U. S. Army Research Office, Research Triangle Park. North Carolina.

Carnegie-Mellon University

Pittsburgh, PA 15213 


\section{PACIFIC JOURNAL OF MATHEMATICS}

\section{EDITORS}

RICHARD ARENS (Managing Editor)

University of California

Los Angeles, California 90024

C. W. Curtis

University of Oregon

Eugene, OR 97403

C. C. MOORE

University of California

Berkeley, CA 94720

\section{J. DUGUNDJI}

Department of Mathematics University of Southern California Los Angeles, California 90007

R. Finn AND J. Milgram Stanford University Stanford, California 94305

ASSOCIATE EDITORS
E. F. BECKENBACH

B. H. NeUMANN

F. WOLF

K. YoshidA

\section{SUPPORTING INSTITUTIONS}

UNIVERSITY OF BRITISH COLUMBIA UNIVERSITY OF SOUTHERN CALIFORNIA CALIFORNIA INSTITUTE OF TECHNOLOGY STANFORD UNIVERSITY UNIVERSITY OF CALIFORNIA MONTANA STATE UNIVERSITY UNIVERSITY OF TOKYO UNIVERSITY OF NEVADA, RENO UNIVERSITY OF UTAH NEW MEXICO STATE UNIVERSITY OREGON STATE UNIVERSITY UNIVERSITY OF OREGON

WASHINGTON STATE UNIVERSITY UNIVERSITY OF WASHINGTON OSAKA UNIVERSITY 


\section{Pacific Journal of Mathematics}

\section{Vol. 74, No. $1 \quad$ May, 1978}

Gerald Arthur Anderson, Computation of the surgery obstruction groups



R. K. Beatson, The degree of monotone approximation ................ 5

Sterling K. Berberian, The character space of the algebra of regulated functions . . . 15

Douglas Michael Campbell and Jack Wayne Lamoreaux, Continua in the plane with

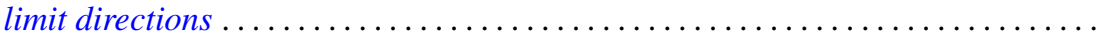

R. J. Duffin, Algorithms for localizing roots of a polynomial and the Pisot



Alessandro Figà-Talamanca and Massimo A. Picardello, Functions that operate on

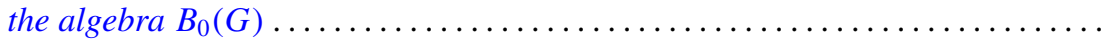

John Erik Fornaess, Biholomorphic mappings between weakly pseudoconvex



Andrzej Granas, Ronald Bernard Guenther and John Walter Lee, On a theorem of S.

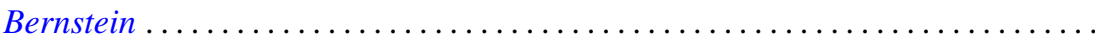

Jerry Grossman, On groups with specified lower central series quotients . .........

William H. Julian, Ray Mines, III and Fred Richman, Algebraic numbers, a constructive development . . . . . . . . . . . . . . . . . . . . . . .

Surjit Singh Khurana, A note on Radon-Nikodým theorem for finitely additive

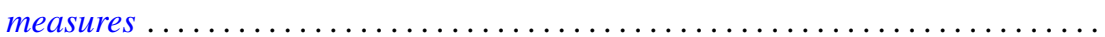

Garo K. Kiremidjian, A Nash-Moser-type implicit function theorem and nonlinear

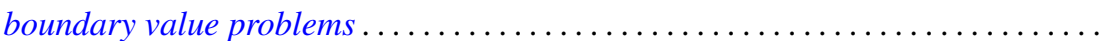

Ronald Jacob Leach, Coefficient estimates for certain multivalent functions ....

John Alan MacBain, Local and global bifurcation from normal eigenvalues. II . . 133

James A. MacDougall and Lowell G. Sweet, Three dimensional homogeneous algebras...

John Rowlay Martin, Fixed point sets of Peano continua ......

R. Daniel Mauldin, The boundedness of the Cantor-Bendixson order of some analytic sets...

Richard C. Metzler, Uniqueness of extensions of positive linear functions ..

Rodney V. Nillsen, Moment sequences obtained from restricted powers . .

Keiji Nishioka, Transcendental constants over the coefficient fields in differential elliptic function fields...

Gabriel Michael Miller Obi, An algebraic closed graph theorem

Richard Cranston Randell, Quotients of complete intersections by $\mathbf{C}^{*}$ actions . . 221

Bruce Reznick, Banach spaces which satisfy linear identities . .

Bennett Setzer, Elliptic curves over complex quadratic fields...

Arne Stray, A scheme for approximating bounded analytic functions on certain subsets of the unit disc.

Nicholas Th. Varopoulos, A remark on functions of bounded mean oscillation and bounded harmonic functions. Addendum to: "BMO functions and the

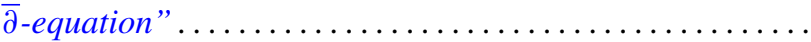

Charles Irvin Vinsonhaler, Torsion free abelian groups quasi-projective over their

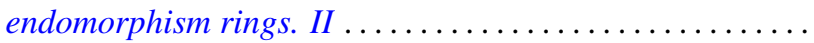

Thomas R. Wolf, Characters of $p^{\prime}$-degree in solvable groups ... 